\title{
Theory for electrochemical impedance spectroscopy of heterogeneous electrode with distributed capacitance and charge transfer resistance
}

\author{
SHWETA DHILLON and RAMA KANT* (1) \\ Complex Systems Group, Department of Chemistry, University of Delhi, Delhi 110 007, India \\ E-mail: rkant@chemistry.du.ac.in
}

MS received 28 January 2017; revised 15 April 2017; accepted 21 April 2017

\begin{abstract}
Randles-Ershler admittance model is extensively used in the modeling of batteries, fuel cells, sensors etc. It is also used in understanding response of the fundamental systems with coupled processes like charge transfer, diffusion, electric double layer charging and uncompensated solution resistance. We generalize phenomenological theory for the Randles-Ershler admittance at the electrode with double layer capacitance and charge transfer heterogeneity, viz., non-uniform double layer capacitance and charge transfer resistance $\left(c_{d}\right.$ and $\left.R_{C T}\right)$. Electrode heterogeneity is modeled through distribution functions of $R_{C T}$ and $c_{d}$, viz., log-normal distribution function. High frequency region captures influence of electric double layer while intermediate frequency region captures influence from the charge transfer resistance of heterogeneous electrode. A heterogeneous electrode with mean charge transfer resistance $\overline{R_{C T}}$ shows faster charge transfer kinetics over a electrode with uniform charge transfer resistance $\left(\overline{R_{C T}}\right)$. It is also observed that a heterogeneous electrode having high mean with large variance in the $R_{C T}$ and $c_{d}$ can behave same as an electrode having low mean with small variance in the $R_{C T}$ and $c_{d}$. The origin of coupling of uncompensated solution resistance (between working and reference electrode) with the charge transfer kinetics is explained. Finally, our model provides a simple route to understand the effect of spatial heterogeneity.
\end{abstract}

Keywords. Randles-Ershler admittance; electrode surface with random charge transfer resistance; ohmic contribution; electric double layer; distribution function for heterogeneity.

\section{Introduction}

Electrochemical impedance spectroscopy (EIS) is a powerful technique for investigating electrochemical systems and processes. Multiple parameters can be determined from a single experiment. EIS can be used to characterize bulk and interfacial properties of the system. It is a high precision measurement technique which does not substantially remove or disturb the system from its operating condition. In real electrochemical systems, impedance spectra are usually more complicated. Randles and Ershler equivalent model ${ }^{1,2}$ is very often used to model the interfacial phenomena and used to characterize EIS of lithium ion batteries, ${ }^{3,4}$ sensors, ${ }^{5,6}$ ion-selective systems ${ }^{7,8}$ and to investigate the stability of films. ${ }^{9}$ It includes ohmic resistance of the electrolyte solution, Warburg impedance (resulting from the diffusion of ions from the bulk electrolyte to the electrode

\footnotetext{
*For correspondence
}

interface), double layer capacitance and electron transfer resistance (that exists if a redox probe is present in the electrolyte solution).

Electrode surface irregularities play significant role in its electrochemical responses. There are several factors which cause these irregularities, like surface roughness corresponding to the geometrical irregularities, chemical heterogeneities (include differences in the constituent materials, chemical imperfections and surface-bound impurities and coatings), atomic-scale inhomogeneities, basal-edge plane heterogeneities, etc. Solid electrodes are not smooth, they exhibit complex surface morphology with the varying degree of irregularities ranging from sub-nanometer to the micrometer length scales. In order to capture the complexity arising from irregular interfaces (i.e., rough, porous, and partially active interfaces) one often uses fractal models ${ }^{10,11}$ and it is the most efficient way to understand the effects of electrode disorder on the electrochemical response. ${ }^{12-14}$ Most of the rough surfaces can be 
approximately mapped into finite self-affine fractals (which exhibit scale invariance over a limited range of the length scales). ${ }^{15,16}$

Along with the electrode roughness, electrochemical properties are also attributed to various heterogeneities; geometric heterogeneity such as roughness or distribution of the pore size and crystallographic disorder (due to anisotropic surface atomic structure). Heterogeneous nanostructured electrode offers high electrochemical energy storage. ${ }^{17}$ The effect of atomic scale heterogeneity is studied on the cyclic voltammetry and time scales are discussed up to which it play significant role in the electrochemical response. ${ }^{18-20}$ Impedance technique is also used to study heterogeneity effects, comparisons are often drawn between the basal and edge plane efficiency. ${ }^{21,22}$ Electrochemical properties over the edge and basal planes are studied, e.g., in single-crystal platinum electrodes in contact with ionic liquids, ${ }^{23}$ on the pyrolytic graphite electrodes ${ }^{24-26}$ or over the graphene sheets. ${ }^{27}$ It has been seen that edge planes are more electrochemically active than the basal planes. $^{23-27}$ The oxygen reduction reaction (ORR) is used in the studies of many electrochemical applications including hydrogen-oxygen fuel cells, biosensors and metal-air batteries. It is found that ORR is prominent on edge as compared to the basal plane on graphite electrode. ${ }^{20,28,29}$ Heterogeneity effects are seen in CPE of impedance at the rough capacitive electrodes, ${ }^{30,31}$ in kinetics over the modified electrodes, monolayers over the electrodes, etc. ${ }^{32,33}$ Contribution of the surface distributions are also studied using finite element simulations. ${ }^{34}$

More than a decade has been spent by Kant and coworkers to understand the impedance behavior at the rough electrodes in presence of various electrochemical processes. Effect of morphological length scales are discussed on the impedance at a finite fractal electrode for a diffusion controlled system. ${ }^{35-37}$ This work has been further extended for the diffusion and homogeneous kinetics coupled with a fast heterogeneous charge transfer reaction ${ }^{38}$ at the realistic fractal electrodes. Influence of quasi-reversible charge transfer, ${ }^{39}$ uncompensated solution resistance ${ }^{40}$ and diffusion limited adsorption coupled to the reversible charge transfer ${ }^{41}$ are also encapsulated to study impedance on the rough and finite fractal electrodes. Randles-Ershler admittance is generalized for the arbitrary topography electrode and is applied to the finite fractal electrodes. ${ }^{42}$ Recently, we have shown that the work function of a metal is dependent on the roughness of surface ${ }^{43}$ whereas work function dependence on the crystallographic phase exposed at the surface is well established. ${ }^{44}$ The roughness and exposure of various crystallographic phases at the electrode surface are random in nature. Hence, the work function of such electrode surfaces are also random in nature. This is also seen in the scanning tunneling microscopy and Kelvin probe force microscopy images of most metal surfaces that they show random distribution of the local work function. ${ }^{45,46}$

Surface heterogeneity and roughness, both significantly control the EIS behavior. Heterogeneity at the disk electrode geometry is widely studied and it is found that ohmic resistance distributions causes the frequency dispersion of impedance measurements. ${ }^{47-49}$ For the disk electrode, Brug et al., developed a model to study the effects of electrode heterogeneity in the double layer capacitance and sluggish charge transfer processes, and showed that the constant phase element (CPE) influences frequency dispersion. ${ }^{50}$ However this model for impedance distribution for CPE with slow charge transfer is good enough when heterogeneity effects are observed in the compact layer and diffusion is unimportant. Recently, Kant et al., extended Brug et al., results in presence of diffuse electric double layer relaxation with CPE and sluggish charge transfer, ${ }^{51}$ and also for the ionic liquids ${ }^{52}$ in presence of roughness. ${ }^{53}$ The objective of this work is to study the influence of electrode heterogeneity on the charge transfer kinetics and capacitance through their statistical overall impedance response.

Figure 1 shows the schematic representation of an electrochemical system with charge transfer and capacitance spatial heterogeneities. Heterogeneous working electrode (WE) with non-uniform charge transfer (site specific) resistance $\left(R_{C T}^{(i)}\right)$ and electric double layer capacitance $\left(c_{d}^{(i)}\right)$ are shown as patches of different colors. For this kind of complex electrochemical system with the multiple interfacial processes, a theory is developed in the next section along with the physical interpretations of various controlling parameters of the interfacial processes. Formulation section is followed by the results and discussions where spatial heterogeneity effects are explained with the help of various impedance plots: Nyquist and Bode's plots. Finally, work is summarized and conclusions are listed.

\section{Formulation}

The conventional Randles-Ershler equivalent circuit area specific impedance is defined as s,2,54 $^{1}$

$z_{R E}(\omega)=R_{s}+\left(j \omega c_{d}+\frac{1}{R_{C T}+z_{W}(\omega)}\right)^{-1}$

where $c_{d}$ is the area specific electric double layer (EDL) capacitance and $z_{W}(\omega)=(\Gamma D \sqrt{j \omega / D})^{-1}$ is the area 


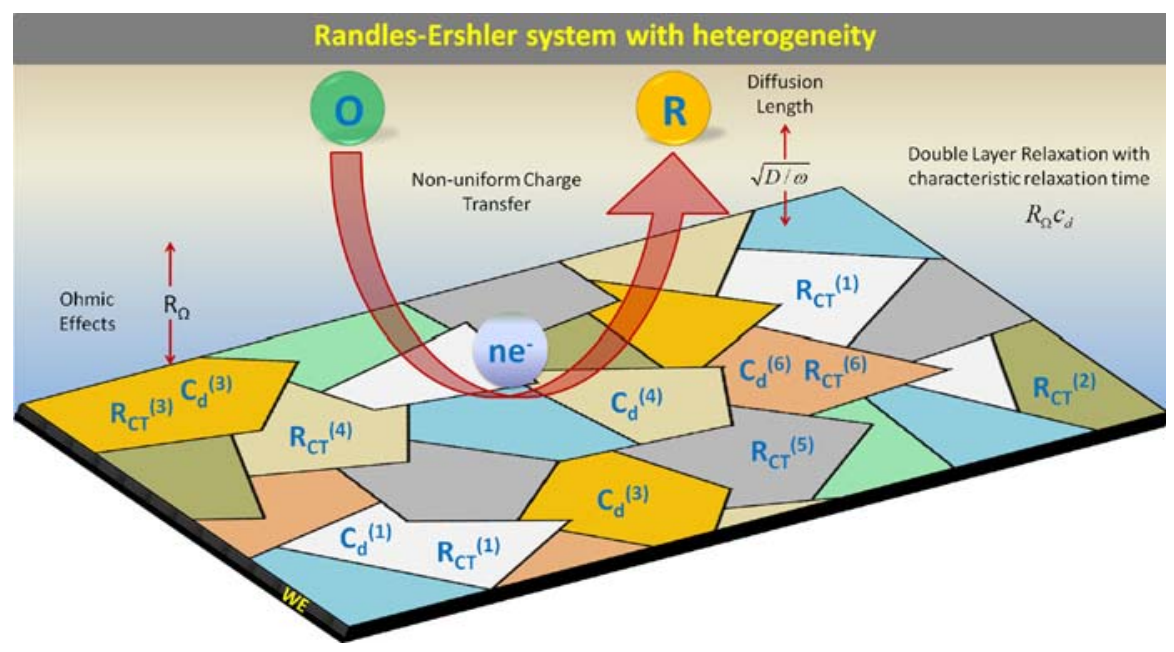

Figure 1. Schematic representation of heterogeneous working electrode (WE) with non-uniform charge transfer (site specific) resistance $\left(R_{C T}^{(i)}\right)$ and double layer capacitance $\left(c_{d}^{(i)}\right)$, and ohmic losses between working (WE) and reference (RE) electrode, $R_{\Omega}$, which influences electrode kinetics. The characteristic electric double layer relaxation time for a patch is $R_{\Omega} c_{d}^{(i)}$.

specific Warburg impedance. $\Gamma=n^{2} F^{2} / R T\left(1 / C_{O}^{0}+\right.$ $\left.1 / C_{R}^{0}\right)$ is the volume specific diffusion capacitance, $n$ is the number of electrons transferred, $F$ is the Faraday constant, $R$ is the gas constant, $T$ is the absolute temperature and $D$ is the diffusion coefficient of electroactive species; $C_{O}^{0}$ and $C_{R}^{0}$ are the bulk concentrations of the oxidized and reduced species, respectively. $R_{C T}$ is the area specific charge transfer resistance while $R_{S}$ is the area specific solution resistance. In equation $1, R_{C T}$ is assumed to be uniform at the electrode surface.

Randles-Ershler equivalent circuit model is extensively used but it works when there are no dynamic interplay of various effects, viz., surface heterogeneity, diffusion, charge transfer, solution resistance and electric double layer charging. But, in the presence of coupling of various phenomena, equivalent circuit model gives erroneous values of various parameters, such as diffusion coefficient, charge transfer resistance, electrolyte resistance, etc. To overcome these limitations of the equivalent circuit analysis, classical Randles-Ershler admittance equation at the smooth and rough surface is developed. ${ }^{42}$ It has dependency on the quasi-reversible charge transfer and solution resistance between the working (WE) and reference (RE) electrode, complex diffusion length and capacitance of the EDL, which are generally present in the broad spectrum impedance experimental data.

Schematic representation of problem of the charge transfer and uncompensated solution resistance, $R_{\Omega}$, (between WE and RE electrode) with the electric double layer charging at the heterogeneous is shown in
Figure 1. The mass transfer diffusion equation is given as: $\frac{\partial \delta C_{\alpha}(\vec{r}, t)}{\partial t}=D_{\alpha} \nabla^{2} \delta C_{\alpha}(\vec{r}, t)$, where $\vec{r}$ is a threedimensional vector $(x, y, z), \alpha$ is $\mathrm{O}$ or $\mathrm{R}$ and it represents the oxidized or reduced species and $D_{\alpha}$ is the diffusion coefficient (for simplicity we assume in our calculations; $\left.D_{O}=D_{R}=D\right)$. $\delta C_{\alpha}(\vec{r}, t)=C_{\alpha}(\vec{r}, t)-C_{\alpha}^{0}$, is difference between surface and bulk concentration of the electroactive species. At an initial time $t=0$, change in concentration of the diffusing species is $\delta C_{\alpha}(\vec{r}, t=0)=0$ and in bulk (far from the electrode), change in concentration of diffusing species is $\delta C_{\alpha}\left(\vec{r}_{\|}, z \rightarrow \infty, t\right)=0$ where $\vec{r}_{\|}$is a two-dimensional vector $(x, y)$. Now, the linearized Butler-Volmer equation along with the capacitive and ohmic contribution can be used to formulate the electrode-electrolyte interface constraint. For the quasi-reversible system in the presence of electric double layer (EDL) capacitance, current at the electrode is determined by the capacitive current of EDL, mass and charge transfer kinetics. The linearized Butler-Volmer equation for small overpotential with EDL capacitive charging in the moderately supported system at the electrode surface, viz. $z=0$ plane ${ }^{42,55,56}$ is (see appendix A for derivation details)

$$
\begin{aligned}
\frac{i\left(\vec{r}_{\|}, t\right)}{i_{0}}= & \frac{\delta C_{O}\left(\vec{r}_{\|}, t\right)}{C_{O}^{0}}-\frac{\delta C_{R}\left(\vec{r}_{\|}, t\right)}{C_{R}^{0}}+n f(\eta(t) \\
& \left.-R_{\Omega} i\left(\vec{r}_{\|}, t\right)\right)-\frac{c_{d}}{i_{0}} \frac{d \eta}{d t}
\end{aligned}
$$

where $i\left(\vec{r}_{\|}, t\right)$ is the local surface profile dependent total current density at the interface and is defined as 
$i\left(\vec{r}_{\|}, t\right)=i_{f}\left(\vec{r}_{\|}, t\right)-c_{d} d \eta(t) / d t, i_{f}\left(\vec{r}_{\|}, t\right)$ is the faradaic component of the current density, $i_{0}$ is the exchange current density and is related to $R_{C T}, i_{0}=R T / n F R_{C T}$, $\eta(t)$ is the applied potential and $c_{d}$ is the area specific EDL capacitance. $f=F / R T$. We wish to determine Randles-Ershler admittance at heterogeneous surface which satisfy various boundary constraints, using fluxbalance condition, $\delta C_{O}\left(\vec{r}_{\|}, t\right)+\delta C_{O}\left(\vec{r}_{\|}, t\right)=0,{ }^{57}$ and assuming same diffusion coefficient for the oxidized and reduced species $\left(D_{O}=D_{R}=D\right)$.

The operative potential at the interface (as shown in the boundary condition, equation 2$)$ is $\eta(t)-R_{\Omega} i\left(\vec{r}_{\|}, t\right)$, where $i\left(\vec{r}_{\|}, t\right)$ is local interfacial current density. So, $R_{\Omega} i\left(\vec{r}_{\|}, t\right)$ is local potential drop between the WE and $\mathrm{RE}$, where $R_{\Omega}$ is the area specific uncompensated solution resistance. The current density, $i\left(\vec{r}_{\|}, t\right)$, arises due to two interfacial phenomena, viz., double layer (non faradaic) charging and faradaic charge transfer current. The multiplicative nature of $R_{\Omega}$ and $i\left(\vec{r}_{\|}, t\right)$ as well as $R_{C T}$ and $i\left(\vec{r}_{\|}, t\right)$ (in equation 2), causes coupling between ohmic $\left(R_{\Omega}\right)$ and charge transfer $\left(R_{C T}\right)$ resistances. So, the emergence of coupling between a bulk property $\left(R_{\Omega}\right)$ and an interfacial property $\left(R_{C T}\right)$ is through the common interfacial current density $\left(i\left(\vec{r}_{\|}, t\right)\right)$. Mathematical origin of this coupling for the linearized surface boundary condition is shown in appendix A. It also shows the additive nature of $R_{C T}$ and $R_{\Omega}$.

Equation 2 can be represented in terms of a phenomenological length scale $\left(L_{C \Omega}\right.$, arising from the charge transfer resistance and ohmic resistance between the WE and RE) and double layer relaxation time scale $\left(\tau_{d l}\right)$. The simplified expression for boundary constraint at the electrode surface is expressed as

$L_{C \Omega} \partial_{n} \delta C_{O}\left(\vec{r}_{\|}\right)-\left.\delta C_{O}\left(\vec{r}_{\|}\right)\right|_{z=0}=\left(1+j \omega \tau_{d l}\right)\left(\frac{\Gamma \eta_{0}}{n F}\right)$

where $\tau_{d l}=c_{d} R_{\Omega} . R_{C T}, R_{\Omega}$ and $R_{C \Omega}$ in combination with diffusion coefficient introduces three characteristic phenomenological length scales, $L_{C T}, L_{\Omega}$ and $L_{C \Omega}$, respectively. $L_{C T}$ and $L_{\Omega}$ are the diffusionkinetics and diffusion-resistance phenomenological lengths, respectively. The composite phenomenological diffusion-kinetics-ohmic length $\left(L_{C \Omega}\right)$ is defined as

$$
\begin{aligned}
& L_{C \Omega}=L_{C T}+L_{\Omega} \\
& L_{C T}=\Gamma D R_{C T} \\
& L_{\Omega}=\Gamma D R_{\Omega}
\end{aligned}
$$

Surprisingly, composite phenomenological length $L_{C \Omega}$ in equation 3 brings coupling of $R_{\Omega}$ and $R_{C T} . L_{C \Omega}$ is the diffusion equilibration layer thickness that arises due to the finite charge kinetics and pseudo-kinetics (due to ohmic contribution in the potential drop at the WE). Hence, the magnitude of $L_{C \Omega}$ will determines the nature of a interfacial kinetics.

If there is fast charge transfer taking place in a fully supported medium, then charge transfer resistance $R_{C T}$ or correspondingly $L_{C T}$ is negligible and the interfacial process is expected to be diffusion controlled. But system with uncompensated resistance, solid electrolyte or low conductivity electrolyte, will show pseudo-kinetic controlled behavior as they will have non-vanishing $L_{C \Omega}$.

For the heterogeneous surface, we can individually solve the Butler-Volmer equation ${ }^{35,39,42}$ for various patches of the charge transfer resistance $\left(R_{C T}^{(i)}\right)$ and double layer capacitance $\left(c_{d}^{(i)}\right)$ and overall admittance response of the heterogeneous electrode is the ensemble average of admittances at all $R_{C T}^{(i)}$ and $c_{d}^{(i)}$. Classical smooth surface Randles-Ershler admittance ${ }^{42}$ (can be seen as admittance over homogeneous charge transfer and double layer capacitance patch), $Y_{R}(\omega)$ is obtained as

$Y_{R}(\omega)=\left(\frac{A_{0}}{y_{W}^{-1}(\omega)+R_{C \Omega}}\right)\left(1+j \omega R_{\Omega} c_{d}\right)$

where $y_{W}(\omega)(=\Gamma D \sqrt{j \omega / D})$ is the area specific Warburg admittance. $R_{C \Omega}$ is defined as $R_{C T}+R_{\Omega}$. $R_{\Omega}$ arises due to separation between the WE and RE (Figure 1). For a conducting electrolyte, smaller is the distance between WE and RE electrodes, smaller is the value of $R_{\Omega}$.

In equation $5, R_{C T}$ and $c_{d}$ are usually assumed to be site independent charge transfer resistance and double layer capacitance, they remain same at the homogeneous electrode surface. Hence, charge transfer resistance and double layer capacitance which are site dependent can be treated as varying charge transfer resistance $\left(R_{C T}^{(i)}\right)$ and electric double layer capacitance $\left(c_{d}^{(i)}\right)$ over various zones of the electrode surface as shown in the Figure 1. It is considered that each patch is large enough so that the patches can be treated as an ensemble of the electrodes with a given $R_{C T}^{(i)}$ and $c_{d}^{(i)}$. Therefore, the whole electrode admittance is considered as an ensemble average of the admittance over various charge transfer resistance and electric double layer capacitance zones. For the fluctuations in the charge transfer resistance and electric double layer capacitance of a heterogeneous surface electrode, $R_{C T}$ can be replaced with $\overline{R_{C T}}+\delta R_{C T}$ while $c_{d}$ can be replaced with $\overline{c_{d}}+\delta c_{d}$ in equation $5 . \overline{R_{C T}}$ and $\overline{c_{d}}$ are the mean value or ensemble average of the charge transfer resistance and electric double layer capacitance, respectively. While $\delta R_{C T}$ and $\delta c_{d}$ are the deviations of the charge transfer resistance and electric double layer capacitance, respectively, from their mean value at a given site. At the lowest level of approximation, $\delta R_{C T}$ 
and $\delta c_{d}$ can be ignored, and $R_{C T}$ can be replaced by $\overline{R_{C T}}$ while $c_{d}$ can be replaced by $\overline{c_{d}}$ (in the equation 5), which can be looked upon as a mean field approximation. Thus, admittance can be written in terms of a mean field Randles-Ershler admittance $\left(\overline{Y_{R}^{0}}(\omega)\right)$ as

$\overline{Y_{R}^{0}}(\omega)=\frac{A_{0}}{y_{W}^{-1}(\omega)+\overline{R_{C \Omega}}}\left(1+j \omega R_{\Omega} \overline{c_{d}}\right)$

where $\overline{R_{C \Omega}}=\overline{R_{C T}}+R_{\Omega}$. Mean field Randles-Ershler admittance $\left(\overline{Y_{R}^{0}}(\omega)\right)$ at the heterogeneous electrode is similar to classical Randles-Ershler admittance $\left(Y_{R}(\omega)\right.$, equation 5) at the homogeneous electrode. Under the condition of lowest approximation, heterogeneous surface behaves like a pseudo-homogeneous surface.

Next step is to account fluctuations in mean field approach for the Randles-Ershler admittance response. This can be done when fluctuations in $R_{C T}$ and $c_{d}$ are small. For small fluctuations in the charge transfer resistance and electric double layer capacitance over a heterogeneous surface, Randles-Ershler admittance can be approximated as (see appendix B for details)

$$
\begin{aligned}
\overline{Y_{R}}(\omega)= & \overline{Y_{R}^{0}}(\omega)\left[1+\frac{\overline{\delta R_{C T}^{2}}}{\left(y_{W}^{-1}(\omega)+\overline{R_{C \Omega}}\right)^{2}}\right. \\
& \left.-\frac{j \omega R_{\Omega} \overline{\delta R_{C T} \delta c_{d}}}{\left(y_{W}^{-1}(\omega)+\overline{R_{C \Omega}}\right)\left(1+j \omega \overline{c_{d}} R_{\Omega}\right)}\right]
\end{aligned}
$$

$\overline{Y_{R}}(\omega)$ is the ensemble averaged Randles-Ershler admittance over all the possible fluctuations in charge transfer resistance over the heterogeneous electrode. $\overline{\delta R_{C T}^{2}}$ is the ensemble average of fluctuations in charge transfer resistance. $\overline{\delta R_{C T} \delta c_{d}}$ is the correlated mean of fluctuations in charge transfer resistance and double layer capacitance. When fluctuations in charge transfer resistance and electric double layer capacitance are uncorrelated, i.e., $\overline{\delta R_{C T}} \overline{\delta c_{d}}$ and $\overline{\delta R_{C T}^{2}} \rightarrow 0$, above equation gives similar result as the classical (equation 5) and mean field (equation 6) Randles-Ershler admittance response. Equation 7 can be simplified for the frequency regime where double layer can be ignored as

$$
\overline{Y_{R}}(\omega) \approx \overline{Y_{R}^{0}}(\omega)\left[1+\left(\frac{y_{W}(\omega)}{1+y_{W}(\omega) \overline{R_{C \Omega}}}\right)^{2} \overline{\delta R_{C T}^{2}}\right]
$$

The characteristic frequency below which electrode will behave as the homogeneous surface electrode is obtained as:

$\omega_{h o} \sim 1 /\left(5 \Gamma^{2} D \overline{\delta R_{C T}^{2}}\right)$

It can be seen that at the low frequency, $\omega<\omega_{\text {ho }}$, heterogeneous Randles-Ershler admittance will be same as the homogeneous Randles-Ershler admittance.
For the high frequency limit $(\omega \rightarrow \infty)$, admittance is controlled by electric double layer capacitance and charge transfer resistance, equation 7 can be approximated as,

$\overline{Y_{R}}(\omega) \approx \overline{Y_{R}^{0}}(\omega)\left(1+\frac{\overline{\delta R_{C T}^{2}}}{\overline{R_{C \Omega}}}-\frac{\overline{\delta R_{C T} \delta c_{d}}}{\overline{c_{d}} R_{\Omega}}\right)$

Equation 10 implies that the characteristic frequency above which the impedance will behave as of homogeneous surface electrode but with constant deviation.

For arbitrary fluctuations which could be large, perturbation theory will break down, hence, it needs to be replaced by the theory with whole distribution function for random charge transfer resistance and electric double layer capacitance. To obtain average response of such an electrode, equation 5 is averaged over charge transfer resistance and electric double layer capacitance distribution functions. As mentioned earlier, the surface distribution of charge transfer resistance and electric double layer capacitance arises due to distribution of work function over the surface. Work function at the electrode surface are random in nature and can be described in the terms of their distribution function. It is fair to assume that most surfaces will show normal distribution of the work function. Charge transfer kinetics is dependent on the work function of the metal and it has been shown that the logarithmic of exchange current density is proportional to the work function. ${ }^{58}$ Hence, surfaces with normal distribution of the work function will have log-normal distribution of the exchange current density or charge transfer resistance. Log-normal distribution function has additional advantage as it does not allow negative values of $R_{C T}$ and $c_{d} \cdot{ }^{59-61}$ Also, due to distribution in the work function, electric double layer capacitance will have different values at different patches of the heterogeneous surface. Hence, the heterogeneous electrode with large charge transfer resistance and electric double layer capacitance fluctuations can be represented through the log-normal distribution function. The probability density function (PDF) of log-normal distribution has the form

$p(x)=\frac{1}{x \sigma \sqrt{2 \pi}} \exp \left(-\frac{1}{2 \sigma^{2}}(\ln x-\mu)^{2}\right)$

where $\mu$ and $\sigma$ are the location parameter of the mean and the scale parameter, respectively. For the log-normal distribution in charge transfer resistance, mean $\left(\overline{R_{C T}}\right)$ is defined as $\exp \left[\mu+\sigma^{2} / 2\right]$ and variance $\left(\sigma_{C T}^{2}\right)$ as $\left(\exp \left[\sigma^{2}\right]-1\right) \exp \left[2 \mu+\sigma^{2}\right]$. The ratio of $\sigma_{C T} / \overline{R_{C T}}$ can be looked upon as the measure of relative spatial heterogeneity in the charge transfer resistance, $\gamma_{c t}$. Similarly for the log-normal distribution in the electric double 
layer capacitance (with $\mu_{d}$ and $\sigma$ as location parameter and the scale parameter, respectively), mean $\left(\overline{c_{d}}\right)$ and variance $\left(\sigma_{d}^{2}\right)$ can be defined as $\exp \left[\mu_{d}+\sigma^{2} / 2\right]$ and $\left(\exp \left[\sigma^{2}\right]-1\right) \exp \left[2 \mu_{d}+\sigma^{2}\right]$, respectively. Relative spatial heterogeneity in the electric double layer capacitance $\left(\gamma_{c}\right)$ in given as the ratio of $\sigma_{d} / \overline{c_{d}}$.

Different patches of charge transfer resistance act as ensemble of the electrodes, hence classical RandlesErshler admittance is averaged over these patches along with their weights from their log-normal distribution. Thus, averaged classical Randles-Ershler admittance equation with the spatial heterogeneity can be written as,

$$
\begin{aligned}
\overline{Y_{R}}(\omega)= & \int_{0}^{\infty} \int_{0}^{\infty} \frac{A_{0}}{y_{W}^{-1}(\omega)+R_{C \Omega}}\left(1+j \omega R_{\Omega} c_{d}\right) \\
& p\left(R_{C T}\right) p\left(c_{d}\right) d R_{C T} d c_{d}
\end{aligned}
$$

where $R_{C \Omega}$ is $R_{C T}+R_{\Omega} \cdot p\left(R_{C T}\right)$ and $p\left(c_{d}\right)$ are the probability distribution of charge transfer resistance and double layer capacitance, respectively. Under the limit of small spatial heterogeneity, perturbation result in the equation 7 and equation 12 will give similar response. For a system with the small current or ohmic resistance, equations 5, 6 and 12 emphasize that the $R_{C T}$ and $R_{\Omega}$ are additive in nature, which was shown rigorously in the earlier works. ${ }^{40,42,55,56}$

\section{Results and Discussion}

Here, we analyze the theoretical results developed for the heterogeneous Randles-Ershler admittance equation but without the surface roughness (equation 12) and compare them with the results of homogeneous electrode (equation 5).

Figure 2 shows the effects of spatial heterogeneity in the charge transfer resistance $\left(R_{C T}\right)$ and the electric double layer capacitance $\left(c_{d}\right)$. Differences in the electrochemical process and their impedance responses are contrasted through three types of graphs: (a) impedance phase vs logarithmic of the frequency (Bode's phase plot), (b) double logarithmic plot of the magnitude of impedance vs logarithmic frequency (Bode's magnitude ploy) and (c) the Nyquist plot (complex-plane impedance diagram). In the Bode's phase plots, phase of the impedance $\left(\Phi=\tan ^{-1}\left(-Z^{\prime \prime} / Z^{\prime}\right)\right)$ is plotted with the logarithmic of the frequency $(\omega)$ while in the Bode's magnitude plots, logarithmic of magnitude of the impedance $(|Z|)$ is plotted with the logarithmic of the frequency $(\omega)$. In the Nyquist plots, real part of the impedance $\left(Z^{\prime}\right)$ is plotted on the $\mathrm{x}$-axis while negative of the imaginary part of the impedance $\left(-Z^{\prime \prime}\right)$ is plotted
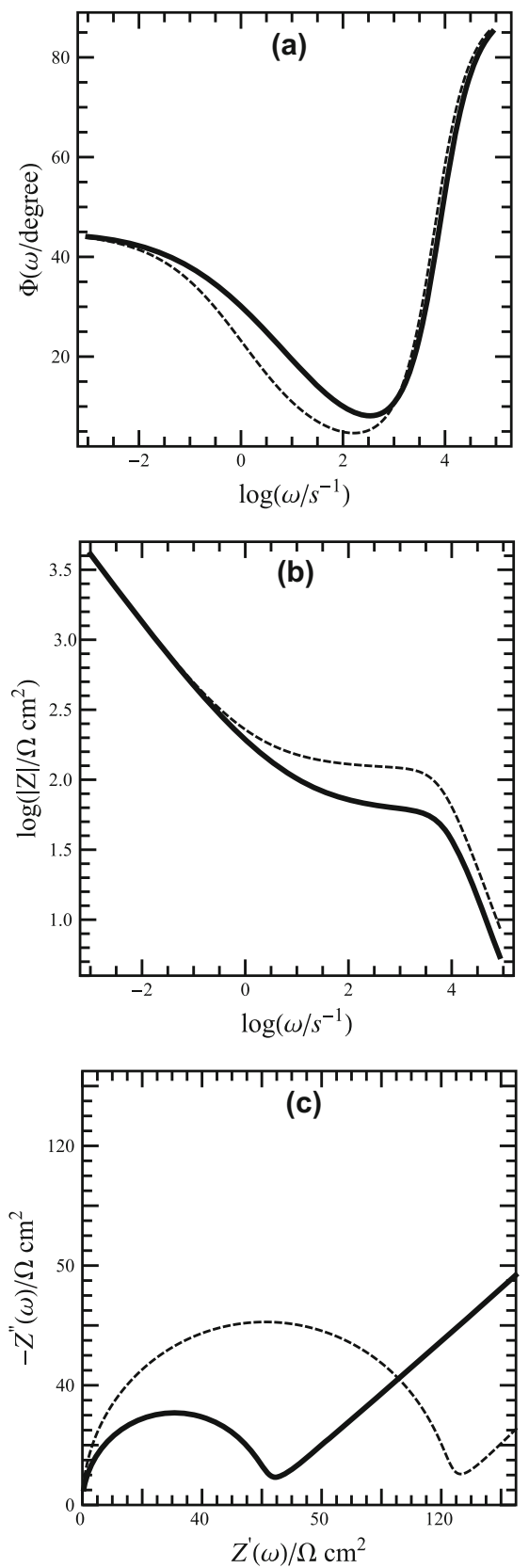

Figure 2. Effect of heterogeneity in charge transfer resistance and electric double layer capacitance is studied using Randles-Ershler impedance on (a) Bode phase angle plot, (b) Bode magnitude plot and (c) Nyquist plot. The dashed line represent classical electrode Randles-Ershler impedance at homogeneous electrode while solid line represent Randles-Ershler impedance at heterogeneous electrode. These plots are generated using $\bar{R}_{C T}=100 \Omega \mathrm{cm}^{2}$ (for $\mu=3.9, \sigma=1.2$ and $\left.\sigma_{C T}=179 \Omega \mathrm{cm}^{2}\right)$, $R_{\Omega}=20 \Omega \mathrm{cm}^{2}, \bar{c}_{d}=8 \mu \mathrm{F} / \mathrm{cm}^{2}$ (for $\mu_{d}$ $=-12.5, \sigma=1.2$ and $\left.\sigma_{d}=14.4 \mu F / \mathrm{cm}^{2}\right)$, $A_{0}=1 \mathrm{~cm}^{2}, D=7 \times 10^{-7} \mathrm{~cm}^{2} / \mathrm{s}, C_{O}^{0}=$ $C_{R}^{0}=5 \mathrm{mM}, n=1$ and $T=298 K$. 

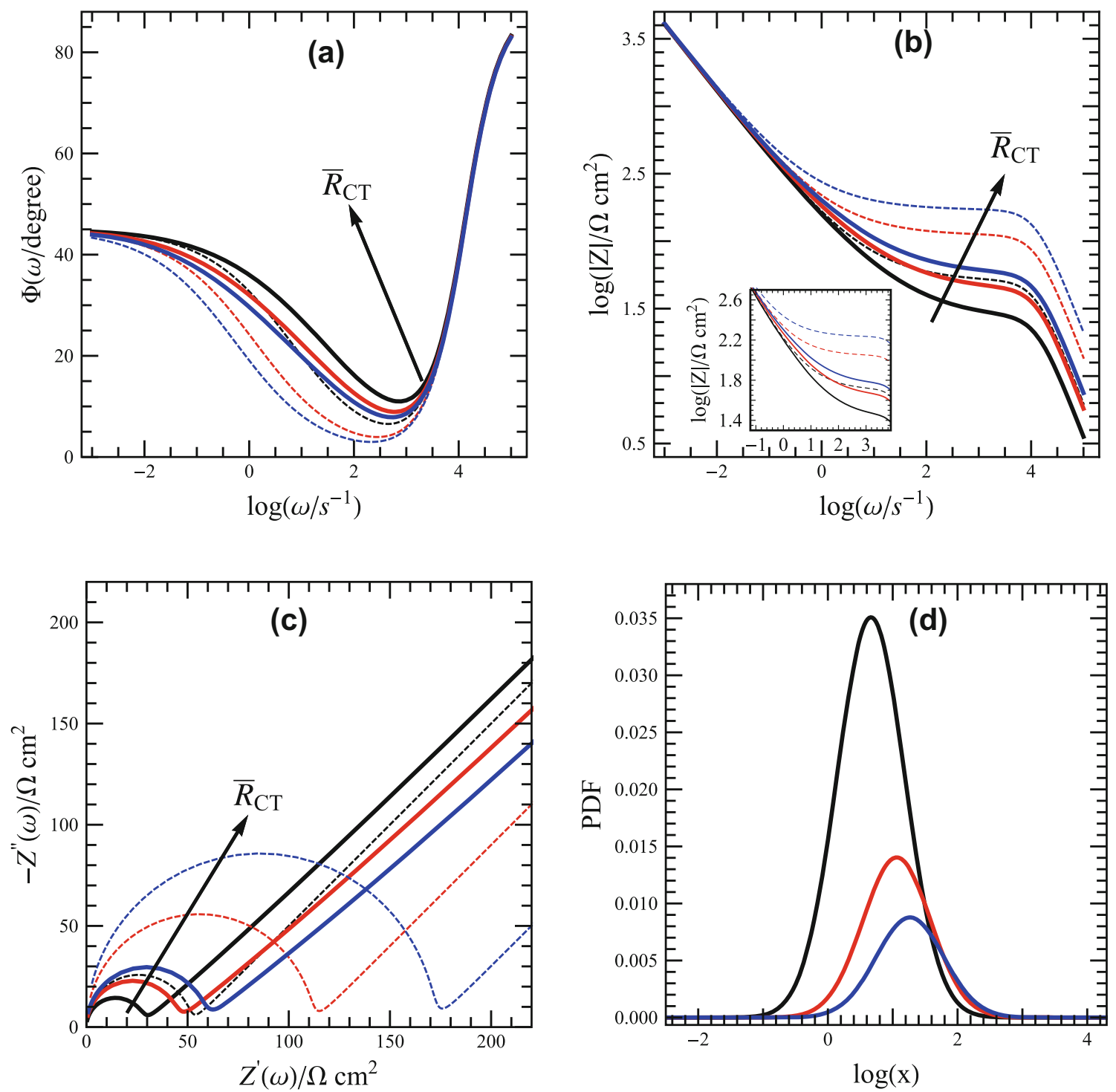

Figure 3. Effect of $\bar{R}_{C T}$ at a surface with fixed electric double layer capacitance $\left(\bar{c}_{d}\right)$ heterogeneity Randles-Ershler impedance on (a) Bode phase angle plot, (b) Bode magnitude plot, (c) Nyquist plot and (d) probability distribution function (PDF). These plots are generated using $R_{\Omega}=10 \Omega \mathrm{cm}^{2}, \bar{c}_{d}=8 \mu \mathrm{F} / \mathrm{cm}^{2}$ (for $\mu_{d}=-12.4, \sigma=1.2$ and $\sigma_{d}=14.4 \mu F / \mathrm{cm}^{2}$ ), $A_{0}=1 \mathrm{~cm}^{2}, D=7 \times 10^{-7} \mathrm{~cm}^{2} / \mathrm{s}, C_{O}^{0}=C_{R}^{0}=5 \mathrm{mM}, n$ $=1$ and $T=298 \mathrm{~K}$. The dashed and solid represent Randles-Ershler impedance response at homogeneous and heterogeneous electrodes, respectively. $\bar{R}_{C T}$ varies as $40 \Omega \mathrm{cm}^{2}$ (for $\mu=3.0, \sigma=1.2$ and $\sigma_{C T}=71.8$ $\Omega \mathrm{cm}^{2}$ ), $100 \Omega \mathrm{cm}^{2}$ (for $\mu=3.9, \sigma=1.2$ and $\sigma_{C T}=179.5 \Omega \mathrm{cm}^{2}$ ) and $160 \Omega \mathrm{cm}^{2}$ (for $\mu=4.4, \sigma=1.2$ and $\sigma_{C T}=287.1 \Omega \mathrm{cm}^{2}$ ) in classical Randles-Ershler impedance equation with heterogeneity for black, red and blue colored lines, respectively.

on the y-axis. Black dashed line represents the classical Randles-Ershler impedance (equation 5) without heterogeneity while black solid line shows the Randles-Ershler impedance (equation 12) curves accounting for the electrode charge transfer heterogeneity. Figure 2(a) shows the phase vs logarithmic of the frequency. Here, low frequency regime is the diffusion controlled as phase $\Phi(\omega) \rightarrow 45^{\circ}$. Minimum in phase is characteristic of $R_{C T}$ and $R_{\Omega}$. On approaching high frequency regime, phase again lifts up due to the charge reorganization, this time due to interaction between the charge transfer and double layer, and phase approaches $90^{\circ}$. Figure 2(b) is the Bode magnitude plot. This magnitude plot is divided into three characteristic frequency regimes: (a) pure Warburg regime, with slope $1 / 2$, (b) charge transfer and ohmic loss (resistance between the WE and RE $\left(R_{\Omega}\right)$ ) controlled regime with slope less than $1 / 2$, and (c) capacitive controlled regime with slope greater than $1 / 2$ and close to 1 . When heterogeneity is introduced, it shows its effect in the intermedi- 

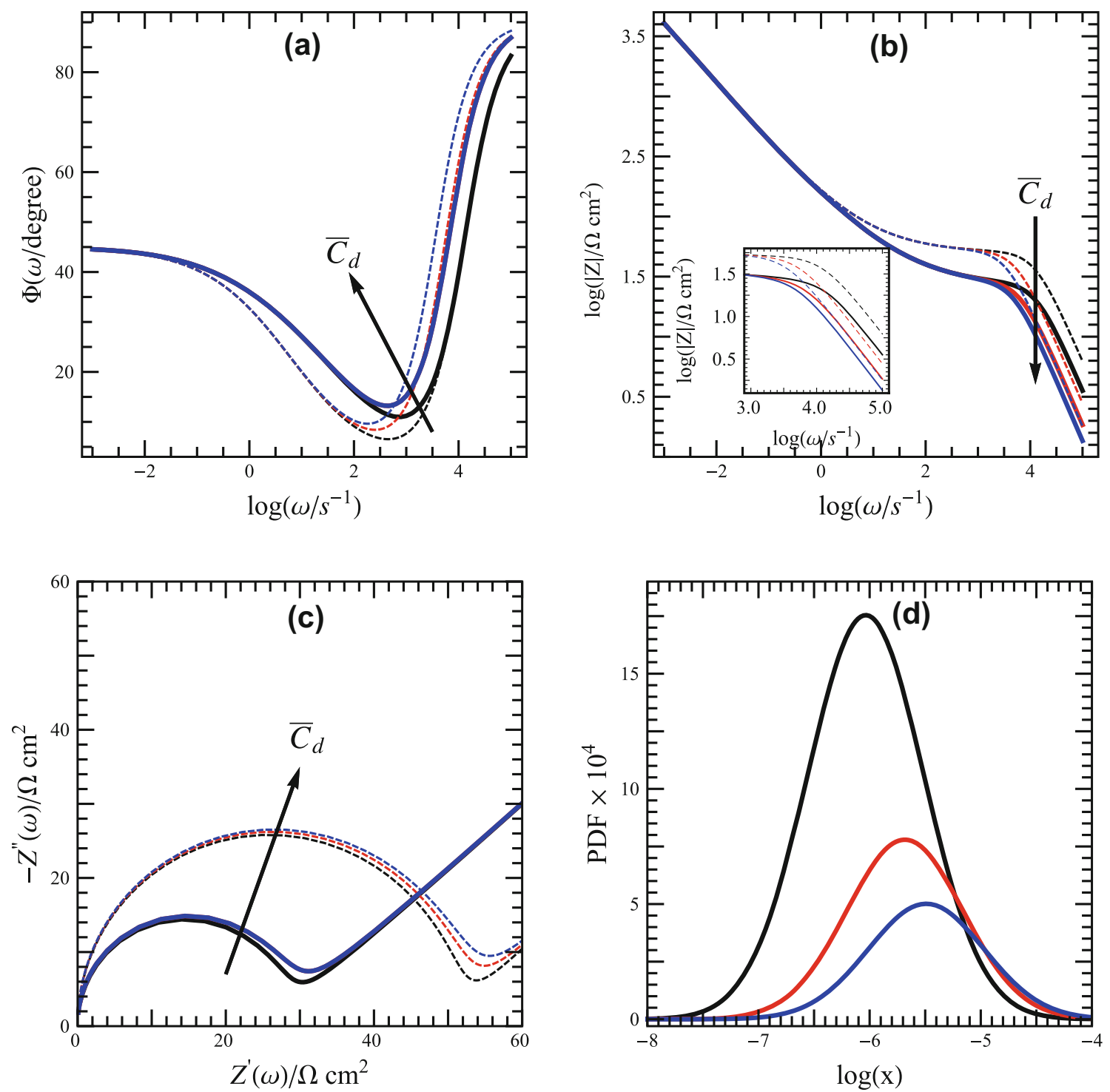

Figure 4. Effect of $\bar{c}_{d}$ at a surface with fixed electric double layer capacitance $\left(\bar{R}_{C T}\right)$ heterogeneity Randles-Ershler impedance on (a) Bode phase angle plot, (b) Bode magnitude plot, (c) Nyquist plot and (d) probability distribution function (PDF). These plots are generated using $R_{\Omega}=10 \Omega \mathrm{cm}^{2}, \bar{R}_{C T}=40 \Omega \mathrm{cm}^{2}$ (for $\mu=3.0, \sigma=1.2$ and $\sigma_{C T}=71.8 \Omega \mathrm{cm}^{2}$ ), $A_{0}=1 \mathrm{~cm}^{2}, D=7 \times 10^{-7} \mathrm{~cm}^{2} / \mathrm{s}, C_{O}^{0}=C_{R}^{0}=5 \mathrm{mM}, n$ $=1$ and $T=298 K$. The dashed and solid represent Randles-Ershler impedance response at homogeneous and heterogeneous electrodes, respectively. $\bar{c}_{d}$ varies as $8 \mu \mathrm{F} / \mathrm{cm}^{2}$ (for $\mu_{d}=-12.5, \sigma=1.2$ and $\sigma_{d}=14.4$ $\mu F / \mathrm{cm}^{2}$ ), $18 \mu F / \mathrm{cm}^{2}$ (for $\mu_{d}=-11.6, \sigma=1.2$ and $\sigma_{d}=32.3 \mu F / \mathrm{cm}^{2}$ ) and $28 \mu F / \mathrm{cm}^{2}$ (for $\mu_{d}=-11.2, \sigma=$ 1.2 and $\left.\sigma_{d}=50.2 \mu \mathrm{F} / \mathrm{cm}^{2}\right)$ in classical Randles-Ershler impedance equation with heterogeneity for black, red and blue colored lines, respectively.

ate regime where impedance response of the classical Randles-Ershler impedance with spatial heterogeneity is lower in magnitude than the classical Randles-Ershler impedance without heterogeneity. Heterogeneity in $R_{C T}$ and $c_{d}$ is evident in the intermediate and high frequency regime. Figure 2(c) shows the effect of heterogeneity in the Nyquist diagram. The impedance curve shows an "inverted hockey stick" shaped response in the Nyquist plot where semicircle in the impedance plot corresponds to the kinetics controlled (charge transfer) and uncompensated solution resistance between the WE and RE $\left(R_{\Omega}\right)$ controlled regime. The raising arm of the curve corresponds to the mass transfer (diffusion) controlled regime. Semicircle in the Nyquist plot is not only characteristic of the kinetics but it can also be seen in absence of electroactive species in the electrolyte. In this case, semicircle arises due to the presence of $R_{\Omega}$ and system shows pseudo-quasi-reversibility. This effect cannot 
be predicted with the equivalent circuit model (equation 1), as equivalent circuit model considers semicircle solely depends upon the charge transfer resistance. When heterogeneity effects are accounted, size of semicircle decreases and knee position in the impedance Nyquist plot shifts to the lower frequency and diffusion controlled regime starts appearing at the shorter frequency.

Figure 3 shows the effect of mean charge transfer resistance $\left(\overline{R_{C T}}\right)$. Solid colored lines represent heterogeneous electrode impedance response while dashed lines represent the corresponding homogeneous electrode impedance response at $\overline{R_{C T}}$ (calculated using $\mu$ and $\sigma)$. In this figure, $\sigma$ is kept constant and $\mu$ is increased (from black to blue). $\overline{R_{C T}}$ and $\sigma_{C T}$ are increased simultaneously which is seen in the Figure 3 . With increase in $\mu$ ( $\overline{R_{C T}}$ increases as $\sigma$ is constant), electrochemical process becomes more sluggish and shifts towards the lower frequency. In Figure 3(a), increase in $\mu$ deviates phase from diffusion type behavior. Peak at the high frequency increases with increase of $\mu$ which pulls process towards more capacitive behavior. At the intermediate regime, minimum shifts towards lower frequency and broadens with increase of $\mu$, implying that the electrochemical process becomes more sluggish. Similar observation can be made from the Figure 3(b). At intermediate regime, with increase of $\mu$, slope of magnitude of impedance decreases and stretches impedance response over broader frequency range. In Figure 3(c), size of the semicircle increases and diffusion controlled regime gets delayed with an increase of heterogeneity however heterogeneous surface shows fast charge transfer kinetics as compared to the homogeneous surface for the same mean charge transfer resistance. Figure 3(d) shows the normalized probability distribution function (PDF) of a log-normal distribution vs logarithmic of the heterogeneous charge transfer resistance (scaled with 1 $\Omega \mathrm{cm}^{2}$ ).

Similarly, Figure 4 shows the effect of mean electric double layer capacitance $\left(\overline{c_{d}}\right) . \sigma$ is kept constant for charge transfer resistance and electric double layer capacitance heterogeneity and $\mu_{d}$ is increased (from black to blue) to increase $\overline{c_{d}}$. Unlike $\overline{R_{C T}}$, effect of $\overline{c_{d}}$ are observed in the high frequency regime (Figure 4(a), (b) and (c)). Effect of distribution of double layer capacitance are seen in the high frequency regime. Various PDF curves of the log-normal distribution of double layer capacitance vs its logarithmic (scaled with $1 \mu \mathrm{F} / \mathrm{cm}^{2}$ ) are shown in the Figure 4(d).

When heterogeneity is increased, i.e., variance in $R_{C T}$ or $c_{d}$ is increased (keeping magnitude of $\sigma$ same for both and increasing $\mu$ or $\mu_{d}$ ), minimum and peak positions shifts towards the higher frequency and phase
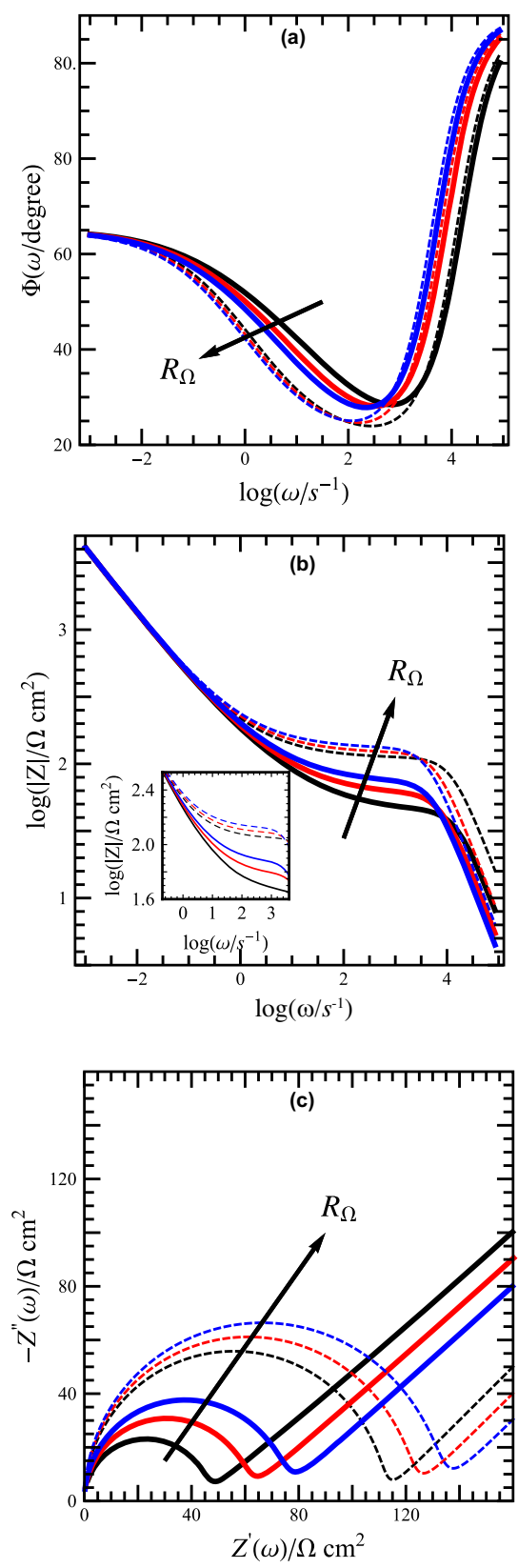

Figure 5. Effect of $R_{\Omega}$ at a surface with fixed heterogeneity using Randles-Ershler impedance on (a) Bode phase angle plot, (b) Bode magnitude plot and (c) Nyquist plot. These plots are generated using $\bar{R}_{C T}=100 \Omega \mathrm{cm}^{2}$ (for $\mu=3.9, \sigma=1.2$ and $\sigma_{C T}=179.5$ $\Omega \mathrm{cm}^{2}$ ), $\bar{c}_{d}=8 \mu \mathrm{F} / \mathrm{cm}^{2}$ (for $\mu_{d}=$ -12.4, $\sigma=1.2$ and $\sigma_{d}=14.4 \mu \mathrm{F} / \mathrm{cm}^{2}$ ), $A_{0}=1 \mathrm{~cm}^{2}, D=7 \times 10^{-7} \mathrm{~cm}^{2} / \mathrm{s}$, $C_{O}^{0}=C_{R}^{0}=5 \mathrm{mM}, n=1$ and $T=$ $298 K$. The dashed and solid represent Randles-Ershler impedance response at homogeneous and heterogeneous electrodes, respectively. $R_{\Omega}$ varies as 10 , 20 and $30 \Omega / \mathrm{cm}^{2}$ in classical Randles-Ershler impedance equation with heterogeneity for black, red and blue colored lines, respectively. 

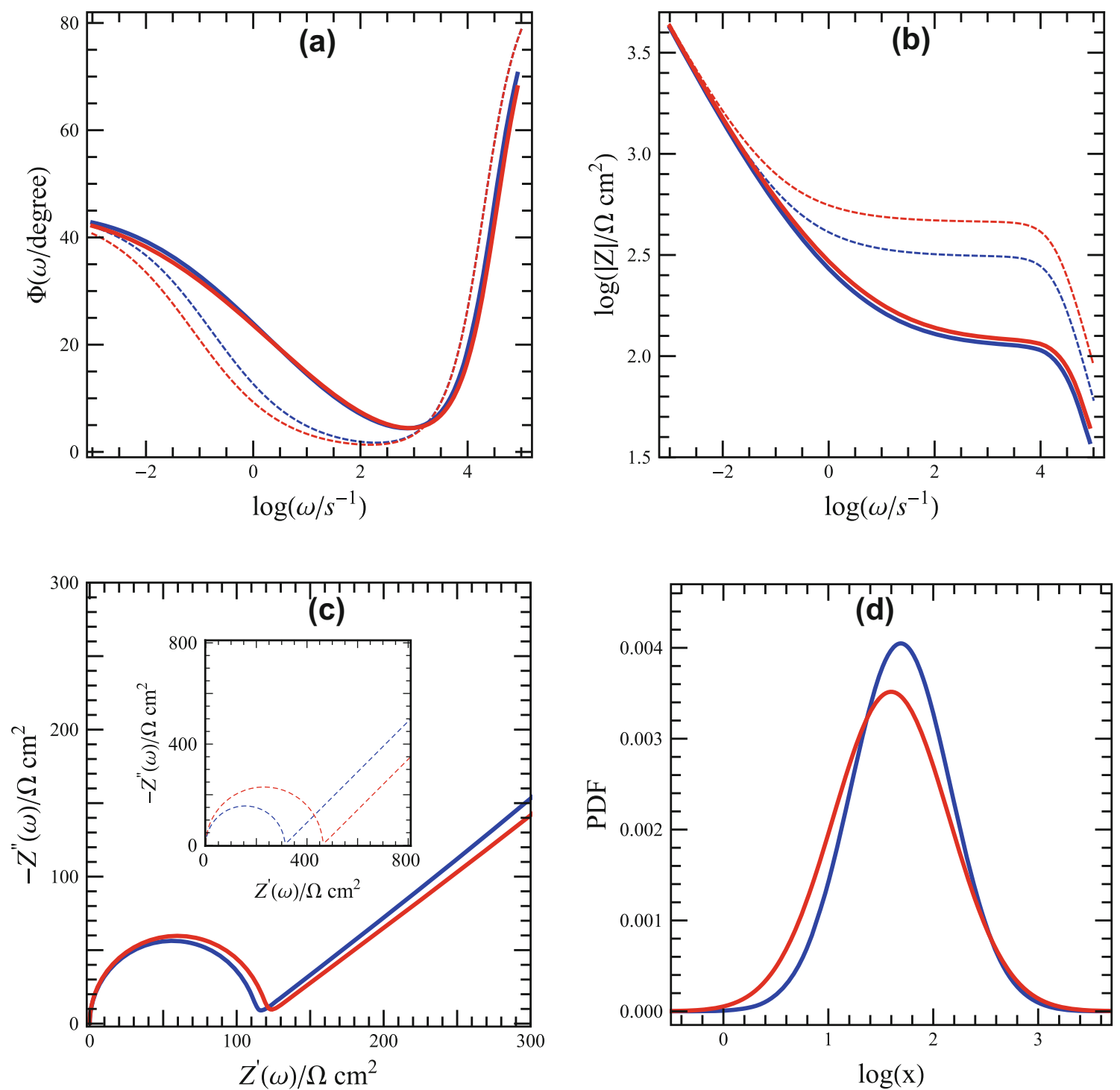

Figure 6. (a) Bode phase angle plot, (b) Bode magnitude plot, (c) Nyquist plot and (d) probability distribution function (PDF) of charge transfer resistance. Blue lines are generated using $\bar{R}_{C T}=300 \Omega \mathrm{cm}^{2}$, (for $\mu$ $=5.1, \sigma=1.1$ and $\sigma_{C T}=459.4 \Omega \mathrm{cm}^{2}$ ) and $\bar{c}_{d}=5 \mu \mathrm{F} / \mathrm{cm}^{2}$ (for $\mu_{d}=-12.8, \sigma=1.2$ and $\sigma_{d}=7.7 \mu F / \mathrm{cm}^{2}$ ). Red lines are generated using $\bar{R}_{C T}=450 \Omega \mathrm{cm}^{2}$ (for $\mu=5.3, \sigma=1.3$ and $\sigma_{C T}=905.1 \Omega \mathrm{cm}^{2}$ ) and $\bar{c}_{d}=5$ $\mu F / \mathrm{cm}^{2}$ (for $\mu_{d}=-13.0, \sigma=1.3$ and $\sigma_{d}=10.1 \mu \mathrm{F} / \mathrm{cm}^{2}$ ). Other parameters used to generated lines are $R_{\Omega}=$ $10 \Omega \mathrm{cm}^{2}, A_{0}=1 \mathrm{~cm}^{2}, D=7 \times 10^{-7} \mathrm{~cm}^{2} / \mathrm{s}, C_{O}^{0}=C_{R}^{0}=5 \mathrm{mM}, n=1$ and $T=298 \mathrm{~K}$. The dashed and solid represent Randles-Ershler impedance response at homogeneous and heterogeneous electrodes, respectively.

goes towards Warburg phase. With increase in $\gamma_{c t}$, number of low as well as high charge transfer resistance sites increases. Hence, the charge transfer process will prefer low resistance sites and kinetics will become faster. Thus, the presence of heterogeneity introduces a new mean for charge transfer resistance which is mean inverse of charge transfer resistance $\left(\overline{R_{C T}^{-1}}\right)$. This average arises out of spatial heterogeneity and introduces two types of characteristic frequencies: 1) due to coupling of charge transfer and diffusion process, $\omega_{h o}^{*}=\frac{D}{\left(\Gamma D\left[1 / \overline{R_{C T}^{-1}}+R_{\Omega}\right]\right)^{2}}$ and 2) due to coupling between double layer capacitance and charge transfer resistance, $\omega_{h i}^{*}=\frac{1}{\overline{c_{d}}\left(1 / \overline{R_{C T}^{-1}}+R_{\Omega}\right)}$. Peak position of phase is seen at $\omega_{h i}^{*}$ while minimum position of phase is obtained as the geometric average of $\omega_{h o}^{*}$ and $\omega_{h i}^{*}$, at the $\omega_{m}=\sqrt{\omega_{h i}^{*} \omega_{h o}^{*}}$. For the case of homogeneous $R_{C T}$ electrode, $1 / \overline{R_{C T}^{-1}}=$ $\overline{R_{C T}}$.

Further, we studied the effect of $R_{\Omega}$ keeping spatial heterogeneity and other parameters constant, shown in 


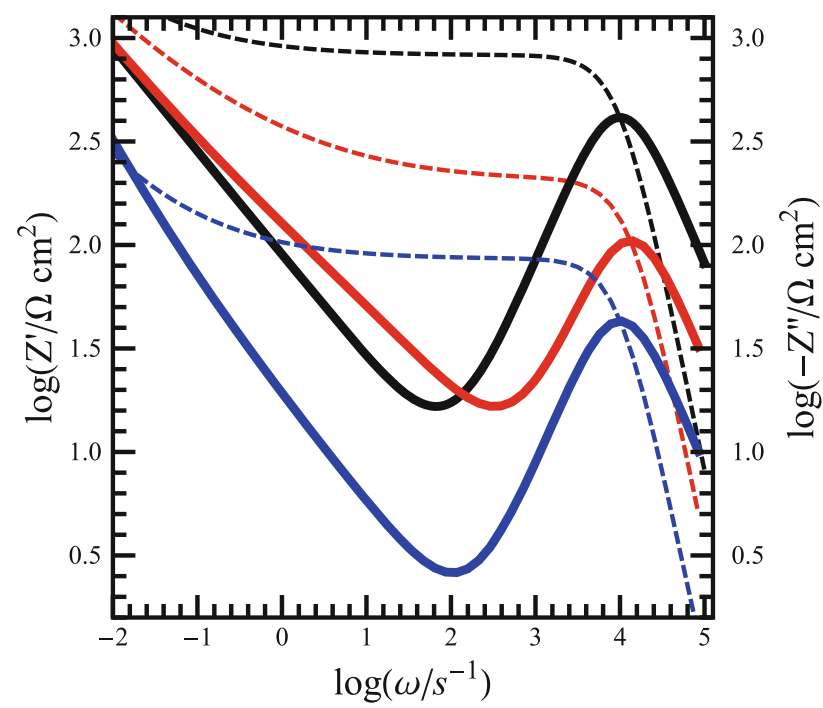

Figure 7. Effect of charge transfer and electric double layer capacitance heterogeneity on real (dashed lines) and imaginary (solid lines) components of impedance are studied. Black and red lines represent Randles-Ershler impedance response at homogeneous and heterogeneous electrodes. Blue line represent the Randles-Ershler impedance at finite fractal electrode (generated using equation 37 of ref. ${ }^{42}$ ). These plots are generated using $\bar{R}_{C T}=814.3 \Omega \mathrm{cm}^{2}$ (for $\mu=5.98, \sigma=$ 1.2 and $\sigma_{C T}=1461.6 \Omega \mathrm{cm}^{2}$ ) and $\bar{c}_{d}=10 \mu \mathrm{F} / \mathrm{cm}^{2}$ (for $\mu_{d}=$ $-12.2, \sigma=1.2$ and $\left.\sigma_{d}=17.9 \mu \mathrm{F} / \mathrm{cm}^{2}\right) R_{\Omega}=10 \Omega \mathrm{cm}^{2}, A_{0}=$ $1 \mathrm{~cm}^{2}, D=7 \times 10^{-7} \mathrm{~cm}^{2} / \mathrm{s}, C_{O}^{0}=C_{R}^{0}=5 \mathrm{mM}, n=1, T=298$ $K, D_{H}$ (fractal dimension $)=2.4, \ell$ (finest size of roughness) $=40 \mathrm{~nm}, l_{\tau}$ (topothesy length) $=0.75 \mu \mathrm{m}$ and $L$ (sample size) $=10 \mu \mathrm{m}$.

the Figure 5. $R_{\Omega}$ depends on the nature of electrolyte and distance between the WE and RE. Usually, it is believed that $R_{C T}$ only contributes to the semicircle of the Nyquist plot. Here, we studied the effect of $R_{\Omega}$ on the charge transfer kinetics. It is seen that $R_{\Omega}$ induces pseudo-kinetics and is analogous to the $\overline{R_{C T}}$ behavior. Figure 5(a) shows the effect of $R_{\Omega}$ on the Bode's phase. Increase in $R_{\Omega}$ shifts diffusion controlled regime further towards low frequency. Figure 5(b) shows that intermediate regime of the Bode's magnitude plot is $R_{\Omega}$ dependent. Increase in $R_{\Omega}$ increases the semicircle size and delays the diffusion regime further, as it can be seen in the Figure 5(c).

In Figure 6(a), (b) and (c), impedance is studied over two different heterogeneous surfaces (Figure 6(d)) using various types of impedance plots. Figure 6(d) shows the probability distribution function (PDF) of a log-normal distribution vs logarithmic of heterogeneous charge transfer resistance. Solid curves represents impedance response at the heterogeneous surface (equation 12) while corresponding dashed lines are generated using mean $\overline{R_{C T}}$ at the homogeneous surface (equation 5 ).
Red and blue curve are representing two different heterogeneous surfaces but their impedance response are very similar (Figure 6(a), (b) and (c)). Figure 6(a) peak and minimum of phase decreases and increases with increase of the spatial heterogeneity, respectively, as charge transfer prefers low resistance sites. In the presence of heterogeneity, charge transfer dominantly takes place from the low resistance sites over the electrode and hence, overall process becomes faster. Therefore, phase becomes more diffusion controlled. This further gets verified from the intermediate regime of the Figure 6(b). Slope of the intermediate regime becomes higher with increase in the spatial heterogeneity and process becomes more diffusion controlled and moves towards the high frequency. Similar effects are seen in Figure 6(a) shows the Nyquist plots for the impedance response in the absence (inset plot) and presence of the spatial heterogeneity. It can be seen from this figure that if heterogeneity is not accounted then it will lead to the erroneous result with apparent charge transfer resistance. Size of the semicircle gets smaller with increase in the heterogeneity.

To study the contrast between heterogeneous and homogeneous Randles-Ershler impedance, logarithmic of real and imaginary impedance response vs. logarithmic of frequency are studied in the Figure 7. Dashed and solid lines represent the real and imaginary impedance response. Black and red lines represent impedance response of the homogeneous and heterogeneous electrode's Randles-Ershler impedance responses. Blue lines represent Randles-Ershler impedance response at the finite-fractal rough electrode at the homogeneous electrode ${ }^{42}$ (see equation 37 in ref. $^{42}$ ). A peak is observed in imaginary part of the classical RandlesErshler impedance and finite-fractal Randles-Ershler impedance response without heterogeneity. Peak is followed by minimum at the lower frequency. When heterogeneity is also accounted in the classical RandlesErshler impedance, minimum position shifts to the lower characteristic frequency, $\omega_{h o}$. Due to charge transfer resistance heterogeneity over the electrode, number of low as well as high charge transfer resistance sites increases. Therefore, preferential charge transfer process takes place through the low charge transfer resistance sites. In Figure 7, shift in the minimum position of imaginary component of impedance response can be taken as the indication of presence of spatial heterogeneity in the electrochemical system. It is clear from this figure that the heterogeneity shifts minimum position to the higher frequency while roughness only shift the magnitude but frequency is not changed. Heterogeneity effects are also seen in real part of classical Randles-Ershler impedance response. Heterogeneity narrow down the 
charge transfer controlled regime (intermediate regime) and make diffusion process shift towards the higher frequency.

\section{Conclusion}

Theory for charge transfer resistance and double layer capacitance heterogeneity effect on the impedance response is developed for the Randles-Ershler model at the heterogeneous electrode. Random charge transfer and double layer capacitance heterogeneity is modeled through a distribution function of charge transfer resistance $\left(R_{C T}\right)$ and double layer capacitance $\left(c_{d}\right)$ over the surface. Systems with narrow range of fluctuations in $R_{C T}$ and $c_{d}$ are expressed through the second order perturbation result for admittance in terms of the mean and variance of $R_{C T}$ and $c_{d}$. While log-normal distribution function is used to mimic the realistic systems (having broad distribution in $R_{C T}$ and $c_{d}$ ). Our theory also includes effects of solution resistance arising due to ohmic contribution between the working (WE) and reference (RE) electrode $\left(R_{\Omega}\right)$, it influences the effective kinetics of charge transfer. The various conclusions drawn from complex plane plots of the mean impedance are:

- Our results show, electrode with the charge transfer heterogeneity (mean charge transfer resistance $\left(\overline{R_{C T}}\right)$ ) results in the faster charge transfer kinetics as compared to the charge transfer kinetics over a homogeneous electrode with $R_{C T}$ equal to $\overline{R_{C T}}$. The reason for this anomaly is the distribution of $R_{C T}$ which causes presence of sites with resistance lower than $\overline{R_{C T}}$. Therefore, dominantly charge transfer occurs through low $R_{C T}$ sites. Hence, heterogeneous electrode shows faster charge transfer as compared to the homogeneous electrode.

- Another anomalous observation is that the heterogeneous electrode with high mean and variance in $R_{C T}$ and $c_{d}$ can give similar impedance response as of the electrode with low mean and variance in $R_{C T}$ and $c_{d}$. This happens due to availability of large number of low charge transfer resistance sites.

- The characteristic low frequency limit beyond which heterogeneity effects are seen is $\omega_{h o} \sim 1$ / $\left(5 \Gamma^{2} D \overline{\delta R_{C T}^{2}}\right)$.

- It is conjunctured that the electrode kinetics of the heterogeneous system is controlled by harmonic mean, viz. inverse of charge transfer resistance $\left(\overline{R_{C T}^{-1}}\right)$, instead of arithmetic mean charge transfer resistance $\overline{R_{C T}}$. For a heterogeneous electrode both harmonic and arithmetic mean are related through inequality, $1 / \overline{R_{C T}^{-1}}<\overline{R_{C T}}$. The characteristic frequency where the influence of heterogeneity is maximum is $\omega_{m}=\sqrt{1 /\left(\overline{c_{d}} \Gamma^{2} D\left(\overline{R_{C T}^{-1}}+R_{\Omega}\right)^{3}\right)}$.

- Heterogeneity causes peak shift to the higher frequency in imaginary impedance response. Hence, the extent of shift in the peak position can be taken as extent of the spatial heterogeneity. Another surface disorder which influences impedance is roughness but it causes shifts in the magnitude of imaginary component while peak position remains unaffected.

- In our theory, it is shown rigorously that $R_{\Omega}$ gets coupled to the $R_{C T}$ via interfacial current density (through ohmic loss potential) and hence, influences effective kinetic parameters of charge transfer. This leads to the scaling of Nyquist impedance semicircles with the higher apparent charge transfer resistance, viz. $R_{C T}+R_{\Omega}$. Traditionally, origin of $R_{\Omega}$ is looked upon as the experimental artefacts in the impedance spectra. But our theory shows, apparent charge transfer resistance obtained from the size of semicircle is combined effect of charge transfer kinetics and uncompensated solution resistance between WE and RE.

- From our theory, low frequency real-axis intercept of impedance semicircles (or cell resistance) is $R_{\Omega}+$ $R_{C T}$.

Finally, random electrode surface heterogeneity is ubiquitous electrode feature therefore these effects are present in most data and also cause of variations in repeated electrochemical measurement. Experimental validity of our methodology can be based on the local electrochemical impedance spectroscopy (LEIS) ${ }^{63-66}$ for systems like glassy carbon electrode ${ }^{67}$ and iron oxide electrode. ${ }^{68}$ LEIS can be used for mapping electrode's impedance distribution which will be useful for understanding the influence of distributed EDL capacitance and charge transfer resistance.

\section{Acknowledgements}

R.K. thanks University of Delhi for the financial support under "Scheme to Strengthen R\&D Doctoral Research Programme". R.K. and S.D. (for SRF fellowship) are grateful to the DST-SERB (Project No. SB/S1/PC-021/2013)-India for providing the financial assistance.

\section{Appendix}

\section{A. Derivation of surface boundary constraint}

The local current density due to diffusional current at an arbitrary position on the electrode surface $(x, y, z=0)$, 
is given by

$i_{f}\left(\vec{r}_{\|}, t\right)=n F D_{O} \partial_{z} \delta C_{O}\left(\vec{r}_{\|}, t\right)$

where $D_{O}$ is diffusion coefficient of the oxidized species and $\partial_{z}=\partial / \partial z$.

Surface boundary condition is derived using the Butler-Volmer equation:

$$
\begin{aligned}
\frac{i_{f}\left(\vec{r}_{\|}, t\right)}{i_{0}}= & \frac{C_{O}\left(\vec{r}_{\|}, t\right)}{C_{O}^{0}} \mathrm{e}^{\alpha n f \eta(t)} \\
& -\frac{C_{R}\left(\vec{r}_{\|}, t\right)}{C_{R}^{0}} \mathrm{e}^{-(1-\alpha) n f \eta(t)}
\end{aligned}
$$

where $i_{f}\left(\vec{r}_{\|}, t\right)$ is the faradaic current density. For the moderately supporting systems and correcting for double layer, above equation can be rewritten as

$$
\begin{aligned}
\frac{i\left(\vec{r}_{\|}, t\right)}{i_{0}}= & \frac{C_{O}\left(\vec{r}_{\|}, t\right)}{C_{O}^{0}} \mathrm{e}^{\alpha n f\left(\eta(t)-i\left(\vec{r}_{\|}, t\right) R_{\Omega}\right)} \\
& -\frac{C_{R}\left(\vec{r}_{\|}, t\right)}{C_{R}^{0}} \mathrm{e}^{-(1-\alpha) n f\left(\eta(t)-i\left(\vec{r}_{\|}, t\right) R_{\Omega}\right)} \\
& -\frac{c_{d}}{i_{0}} \frac{d \eta}{d t}
\end{aligned}
$$

where $i\left(\vec{r}_{\|}, t\right)=i_{f}\left(\vec{r}_{\|}, t\right)-c_{d} \frac{d \eta(t)}{d t}$ is the combination of both faradaic and non-faradaic component of current density. ${ }^{62}$ Replacing $C_{m}\left(\vec{r}_{\|}, t\right)$ by $\delta C_{m}\left(\vec{r}_{\|}, t\right)+C_{m}^{0}$, where $m=O$ or $R$

$$
\begin{aligned}
\frac{i\left(\vec{r}_{\|}, t\right)}{i_{0}}= & \frac{\delta C_{O}\left(\vec{r}_{\|}, t\right)}{C_{O}^{0}} \mathrm{e}^{\alpha n f\left(\eta(t)-i\left(\vec{r}_{\|}, t\right) R_{\Omega}\right)} \\
& +\mathrm{e}^{\alpha n f\left(\eta(t)-i\left(\vec{r}_{\|}, t\right) R_{\Omega}\right)} \\
& -\frac{\delta C_{R}\left(\vec{r}_{\|}, t\right)}{C_{R}^{0}} \mathrm{e}^{-(1-\alpha) n f\left(\eta(t)-i\left(\vec{r}_{\|}, t\right) R_{\Omega}\right)} \\
& -\mathrm{e}^{-(1-\alpha) n f\left(\eta(t)-i\left(\vec{r}_{\|}, t\right) R_{\Omega}\right)}-\frac{c_{d}}{i_{0}} \frac{d \eta}{d t}
\end{aligned}
$$

For the small external perturbation potential and therefore, small output current density, we can linearize the Butler-Volmer equation,

$$
\begin{aligned}
\frac{i\left(\vec{r}_{\|}, t\right)}{i_{0}}= & \frac{\delta C_{O}\left(\vec{r}_{\|}, t\right)}{C_{O}^{0}}\left(1+\alpha n f \eta(t)-\alpha n f i\left(\vec{r}_{\|}, t\right) R_{\Omega}\right) \\
& +\left(1+\alpha n f \eta(t)-\alpha n f i\left(\vec{r}_{\|}, t\right) R_{\Omega}\right) \\
& -\frac{\delta C_{R}\left(\vec{r}_{\|}, t\right)}{C_{R}^{0}}(1-n f \eta(t)+\alpha n f \eta(t) \\
& \left.+n f i\left(\vec{r}_{\|}, t\right) R_{\Omega}-\alpha n f i\left(\vec{r}_{\|}, t\right) R_{\Omega}\right) \\
& -\left(1-n f \eta(t)+\alpha n f \eta(t)+n f i\left(\vec{r}_{\|}, t\right) R_{\Omega}\right. \\
& \left.-\alpha n f i\left(\vec{r}_{\|}, t\right) R_{\Omega}\right)-\frac{c_{d}}{i_{0}} \frac{d \eta}{d t}
\end{aligned}
$$

Neglecting higher order terms,

$$
\begin{aligned}
\frac{i\left(\vec{r}_{\|}, t\right)}{i_{0}}= & \frac{\delta C_{O}\left(\vec{r}_{\|}, t\right)}{C_{O}^{0}}-\frac{\delta C_{R}\left(\vec{r}_{\|}, t\right)}{C_{R}^{0}}+n f \eta(t) \\
& -n f i\left(\vec{r}_{\|}, t\right) R_{\Omega}-\frac{c_{d}}{i_{0}} \frac{d \eta}{d t}
\end{aligned}
$$

Using the flux-balance condition and assuming that the ions have same diffusion coefficient $\left(D_{O}=D_{R}=\right.$ $D)$, we have concentration constrains on the oxidized and reduced species as ${ }^{57} \delta C_{O}\left(\vec{r}_{\|}, t\right)+\delta C_{R}\left(\vec{r}_{\|}, t\right)=0$. It is assumed that the concentration $\delta C_{O}\left(\vec{r}_{\|}, t\right)$ has oscillatory time dependence $\mathrm{e}^{j \omega t} \delta C_{O}\left(\vec{r}_{\|}\right)$(behaves like the applied potential). For a small sinusoidal applied interfacial potential $\eta(t)=\eta_{0} \exp (j \omega t)$, using $i_{0}=$ $R T /\left(n F R_{C T}\right)$ and $i_{f}\left(\vec{r}_{\|}, t\right)$ from equation A.1 in the equation A.6,

$$
\begin{aligned}
& \frac{n F D_{O} \partial_{z} \delta C_{O}\left(\vec{r}_{\|}\right) \mathrm{e}^{j \omega t}-j \omega c_{d} \eta_{0} e^{j \omega t}}{R T /\left(n F R_{C T}\right)} \\
& =\frac{\delta C_{O}\left(\vec{r}_{\|}\right) \mathrm{e}^{j \omega t}}{C_{O}^{0}}+\frac{\delta C_{O}\left(\vec{r}_{\|}\right) \mathrm{e}^{j \omega t}}{C_{R}^{0}} \\
& \quad+n f\left[\eta_{0} \mathrm{e}^{j \omega t}-R_{\Omega}\left(n f D \partial_{z} \delta C_{O}\left(\vec{r}_{\|}, t\right)\right.\right. \\
& \left.\left.-c_{d} \eta_{0} j \omega \mathrm{e}^{j \omega t}\right)\right] \\
& -n f c_{d} R_{C T} j \omega \eta_{0} \mathrm{e}^{j \omega t}
\end{aligned}
$$

On rearranging the terms, we get

$$
\begin{aligned}
& \frac{n^{2} F^{2}}{R T} D\left(R_{C T}+R_{\Omega}\right) \partial_{z} \delta C_{O}\left(\vec{r}_{\|}\right) \\
&= \delta C_{O}\left(\vec{r}_{\|}\right)\left(\frac{1}{C_{O}^{0}}+\frac{1}{C_{R}^{0}}\right) \\
&+n f \eta_{0} \mathrm{e}^{j \omega t}\left(1+j \omega c_{d}\left(R_{\Omega}+R_{C T}\right)\right) \\
& \quad-n f c_{d} R_{C T} j \omega \eta_{0} \mathrm{e}^{j \omega t}
\end{aligned}
$$

$R_{C T}+R_{\Omega}$ can be written as $R_{C \Omega}$ and $n^{2} F^{2} / R T\left(1 / C_{O}^{0}+\right.$ $\left.1 / C_{R}^{0}\right)$ as $\Gamma$, hence the above equation can be rewritten as

$$
L_{C \Omega} \partial_{z} \delta C_{O}\left(\vec{r}_{\|}\right)-\left.\delta C_{O}\left(\vec{r}_{\|}\right)\right|_{z=0}=\left(1+j \omega \tau_{d l}\right)\left(\frac{\Gamma \eta_{0}}{n F}\right)
$$

where double layer relaxation time is $\tau_{d l}=R_{\Omega} c_{d}$. $L_{C \Omega}$ is phenomenological kinetics-ohmic length is defined as

$$
\begin{aligned}
& L_{C \Omega}=L_{C T}+L_{\Omega} \\
& L_{C T}=\Gamma D R_{C T} \\
& L_{\Omega}=\Gamma D R_{\Omega}
\end{aligned}
$$




\section{B. Small $R_{C T}$ and $c_{d}$ fluctuation approximation}

Replacing local $R_{C T}$ in equation 5 by $\overline{R_{C T}}+\delta R_{C T}$ and $c_{d}$ by $\overline{c_{d}}+\delta c_{d}$, it becomes

$$
\begin{aligned}
Y_{R}(\omega)= & \left(\frac{A_{0}}{y_{W}(\omega)^{-1}+\overline{R_{C T}}+\delta R_{C T}+R_{\Omega}}\right) \\
& \left(1+j \omega R_{\Omega}\left(\overline{c_{d}}+\delta c_{d}\right)\right)
\end{aligned}
$$

above equation can be written as,

$$
\begin{aligned}
Y_{R}(\omega)= & \frac{A_{0}}{y_{W}^{-1}(\omega)+\overline{R_{C \Omega}}+\delta R_{C T}} \\
& \left(1+\overline{c_{d}} j \omega R_{\Omega}+j \omega \delta c_{d} R_{\Omega}\right)
\end{aligned}
$$

where $\overline{R_{C \Omega}}=\overline{R_{C T}}+R_{\Omega}$. On rearranging the terms, we get

$$
\begin{aligned}
Y_{R}(\omega)= & \frac{A_{0}\left(1+j \omega c_{d} \overline{R_{C \Omega}}\right)}{\left(y_{W}^{-1}(\omega)+\overline{R_{C \Omega}}\right)\left(1+\frac{\delta R_{C T}}{y_{W}^{-1}(\omega)+\overline{R_{C \Omega}}}\right)} \\
& \left(1+\frac{j \omega \delta c_{d} R_{\Omega}}{1+j \omega \overline{c_{d}} R_{\Omega}}\right)
\end{aligned}
$$

On expanding and ensemble averaging admittance responses over surface heterogeneity, fluctuations in charge transfer resistance and electric double layer capacitance (are dependent on each other) at the electrode surface, the classical Randles-Ershler admittance for small heterogeneity can be written as

$$
\begin{aligned}
\overline{Y_{R}}(\omega)= & \overline{Y_{R}^{0}}(\omega)\left[1+\frac{\overline{\delta R_{C T}^{2}}}{\left(y_{W}^{-1}(\omega)+\overline{R_{C \Omega}}\right)^{2}}\right. \\
& \left.-\left(\frac{j \omega R_{\Omega} \overline{\delta R_{C T} \delta c_{d}}}{\left(y_{W}^{-1}(\omega)+\overline{R_{C \Omega}}\right)\left(1+j \omega \overline{c_{d}} R_{\Omega}\right)}\right)\right]
\end{aligned}
$$

\section{References}

1. Randles J E B 1947 Kinetics of Rapid Electrode Reactions Disc. Far. Soc. 111

2. Ershler B 1947 Investigation of Electrode Reactions by the Method of Charging-Curves and with the aid of Alternating Currents Disc. Far. Soc. 1269

3. Dokko K, Mohamedi M, Fujita Y, Itoh T, Nishizawa M, Umeda M and Uchida I 2001 Kinetic Characterization of Single Particles of $\mathrm{LiCoO}_{2}$ by $\mathrm{AC}$ Impedance and Potential Step Methods J. Electrochem. Soc. 148 A422

4. Takahashi M, Tobishima S, Takei K and Sakurai Y 2002 Reaction Behavior of $\mathrm{LiFePO}_{4}$ as a Cathode Material for Rechargeable Lithium Batteries Solid State Ionics. 148 283
5. Lisdat F and Schäfer D 2008 The Use of Electrochemical Impedance Spectroscopy for Biosensing Anal. Bioanal. Chem. 3911555

6. Katz E and Willner I 2003 Probing Biomolecular Interactions at Conductive and Semiconductive Surfaces by Impedance Spectroscopy: Routes to Impedimetric Immunosensors, DNA-Sensors, and Enzyme Biosensors Electroanalysis. 15913

7. Nikonenko V and Kozmai A 2012 Electrical Equivalent Circuit of an Ion-Exchange Membrane System Electrochim. Acta $\mathbf{5 6} 1262$

8. Moya A A 2014 Electrochemical Impedance of IonExchange Membranes in Ternary Solutions with Two Counterions J. Phys. Chem. C. 1182539

9. Harris J J and Bruening M L 2000 Electrochemical and in Situ Ellipsometric Investigation of the Permeability and Stability of Layered Polyelectrolyte Films Langmuir. 16 2006

10. Mandelbrot B B 1977 In The Fractal Geometry of Nature (San Francisco: Freeman)

11. Feder J 1988 In Fractals (New York: Plenum)

12. Kant R 1993 Can Current Transients be Affected by the Morphology of the Non fractal Electrode? Phys. Rev. Lett. 704094

13. Kant R 1994 Can One Electrochemically Measure the Statistical Morphology of a Rough Electrode? J. Phys. Chem. 981663

14. Kant R and Rangarajan S K 1994 Effect of Surface Roughness on Diffusion-Limited Charge Transfer $J$. Electroanal. Chem. 3681

15. Srivastav S, Dhillon S, Kumar R and Kant R 2013 Experimental Validation of Roughness Power Spectrum Based Theory of Anomalous Cottrell Response J. Phys. Chem. C 1178594

16. Dhillon S and Kant R 2013 SEM-CV Hybrid Method for 3D-Roughness Characterization, Reconstruction and its Electroactivity Appl. Surf. Sci. 282105

17. Liu R, Duay J and Lee S B 2011 Heterogeneous Nanostructured Electrode Materials for Electrochemical Energy Storage Chem. Commun. 471384

18. Eftekhari A, Kazemzad M and Keyanpour-Rad M 2005 Influence of Atomic-Scale Irregularities in Fractal Analysis of Electrode Surfaces Appl. Surf. Sci. 239311

19. Davies T J, Moore R M, Banks C E and Compton R G 2004 The Cyclic Voltammetric Response of Electrochemically Heterogeneous Surfaces J. Electroanal. Chem. 574123

20. Davies T J, Hyde M E and Comptom R G 2005 Nanotrench Arrays Reveal Insight into Graphite Electrochemistry Angew. Chem. Int. Ed. 445121

21. Janek R P and Fawcett W R 1998 Impedance Spectroscopy of Self-Assembled Monolayers on $\mathrm{Au}(111)$ : Sodium Ferrocyanide Charge Transfer at Modified Electrodes Langmuir 143011

22. Los P, Lasia A, Fournier J, Brossard L and Ménard H 1994 AC Impedance Studies of Highly Oriented Pyrolytic Graphite in $1 \mathrm{M} \mathrm{NaOH}$ Solution J. Electrochem. Soc. 1412716

23. Navarro-Suárez A M, Hidalgo-Acosta J C, Fadini L, Feliu J M and Suárez-Herrera M F 2011 Electrochemical 
Oxidation of Hydrogen on Basal Plane Platinum Electrodes in Imidazolium Ionic Liquids J. Phys. Chem. C 11511147

24. Edwards M A, Bertoncello P and Unwin P R 2009 Slow Diffusion Reveals the Intrinsic Electrochemical Activity of Basal Plane Highly Oriented Pyrolytic Graphite Electrodes J. Phys. Chem. C 1139218

25. Neumann C C M, Batchelor-McAuley C, Downing C and Compton R G 2011 Anthraquinone Monosulfonate Adsorbed on Graphite Shows Two Very Different Rates of Electron Transfer: Surface Heterogeneity Due to Basal and Edge Plane Sites Chem. Eur. J. 177320

26. Banks C E, Moore R R, Davies T J and Compton R G 2004 Investigation of Modified Basal Plane Pyrolytic Graphite Electrodes: Definitive Evidence for the Electrocatalytic Properties of the ends of Carbon Nanotubes Chem. Commun. 161804

27. Yuan W, Zhou Y, Li Y, Li C, Peng H, Zhang J, Liu Z, Dai L and Sh G 2013 The Edge- and Basal-plane-specific Electrochemistry of a Single-Layer Graphene sheet Sci. Rep. 32248

28. Shen A, Zou Y, Wang Q, Dryfe R A W, Huang X, Dou S, Dai L and Shuangyin W 2014 Oxygen Reduction Reaction in a Droplet on Graphite: Direct Evidence that the Edge Is More Active than the Basal Plane Angew. Chem. Int. Ed. 5310804

29. Randviir E P and Banks C E 2014 The Oxygen Reduction Reaction at Graphene Modified Electrodes Electroanalysis $\mathbf{2 6} 736$

30. Kerner Z and Pajkossy T 1998 Impedance of Rough Capacitive Electrodes: the Role of Surface Disorder $J$. Electroanal. Chem. 448139

31. Kerner Z and Pajkossy T 2000 On the Origin of Capacitance Dispersion of Rough Electrodes Electrochim. Acta 46207

32. Sabatani E, Cohen-Boulakia J, Bruening M and Rubinstein I 1993 Thioaromatic Monolayers on Gold: A New Family of Self -Assembling Monolayers Langmuir 9 2974

33. da Rocha J R C, Demets G J, Bertotti M, Araki K and Toma H E 2002 Charge Transfer at Electrostatically Assembled Tetraruthenated Porphyrin Modified Electrodes J. Electroanal. Chem. $\mathbf{5 2 6} 69$

34. Alexander C L, Tribollet B and Orazem M E 2015 Contribution of Surface Distributions to Constant-PhaseElement (CPE) Behavior: 1. Influence of Roughness Electrochim. Acta 173416

35. Kant R and Rangarajan S K 2003 Effect of Surface Roughness on Interfacial Reaction-Diffusion Admittance J. Electroanal. Chem. 552141

36. Kant R, Kumar R and Yadav V K 2008 Theory of Anomalous Diffusion Impedance on Realistic Fractal Electrodes J. Phys. Chem. C Lett. 1124019

37. Kumar R and Kant R 2009 Generalized Warburg Impedance on Realistic Self-affine Fractals: Comparative Study of Statistically Corrugated and Isotropic Roughness J. Chem. Sci. 56579

38. Kumar R and Kant R 2009 Theory of Generalized Gerischer Admittance of Realistic Fractal Electrode $J$. Phys. Chem. C113 19558
39. Kumar R and Kant R 2011 Theory of Quasi-Reversible Charge Transfer Admittance on Finite Self-Affine Fractal Electrode Electrochim. Acta $\mathbf{5 6} 7112$

40. Srivastav S and Kant R 2011 Anomalous Warburg Impedance: Influence of Uncompensated Solution Resistance J. Phys. Chem. C 11512232

41. Kumar R and Kant R 2013 Admittance of Diffusion Limited Adsorption Coupled to Reversible Charge Transfer on Rough and Finite Fractal Electrodes Electrochim. Acta 95275

42. Kant R and Singh M B 2015 Generalization of RandlesErshler Admittance for Arbitrary Topography Electrode: Application to Random Finite Fractal Roughness Electrochim. Acta 163310

43. Kaur J and Kant R 2015 Curvature-Induced Anomalous Enhancement in the Work Function of Nanostructures $J$. Phys. Chem. Lett. 62870

44. Smoluchowski R 1941 Anisotropy of the Electronic Work Function of Metals Phys. Rev. 60661

45. Jia J F, Inoue K, Hasegawa Y, Yang W S and Sakurai T 1998 Variation of the Local Work Function at Steps on Metal Surfaces Studied with STM Phys. Rev. B 581193

46. Sohn A, Kanki T, Sakai K, Tanaka H and Kim D 2015 Fractal Nature of Metallic and Insulating Domain Configurations in a $\mathrm{VO}_{2}$ Thin Film Revealed by Kelvin Probe Force Microscopy Sci. Rep. 510417

47. Newman J 1970 Frequency Dispersion in Capacity Measurements at a Disk Electrode J. Electrochem. Soc. 117 198

48. Huang V M, Vivier V, Orazem M E, Pébère N and Tribollet B 2007 The Apparent Constant-Phase-Element Behavior of a Disk Electrode with Faradaic Reactions. A Global and Local Impedance Analysis J. Electrochem. Soc. 154 C99

49. Alexander C L, Tribollet B and Orazem M E 2016 Influence of Micrometric-Scale Electrode Heterogeneity on Electrochemical Impedance Spectroscopy Electrochim. Acta 201374

50. Brug G J, van den Eeden A L G, Sluyters-Rehbach M and Sluyters J H 1984 The Analysis of Electrode Impedances Complicated by the Presence of a Constant Phase Element J. Electroanal. Chem. Interfacial Electrochem. 176 275

51. Singh M B and Kant R 2013 Debye-Falkenhagen Dynamics of Electric Double Layer in Presence of Electrode Heterogeneities J. Electroanal. Chem. 704197

52. Singh M B and Kant R 2014 Theory of Anomalous Electric Double Layer Dynamics in Ionic Liquids J. Phys. Chem. C118 8766

53. Singh M B and Kant R 2014 Theory of Anomalous Dynamics of Electric Double Layer at Heterogeneous and Rough Electrodes J. Phys. Chem. C 1185122

54. Grahame D C 1952 Mathematical Theory of the Faradaic Admittance: Pseudocapacity and Polarization Resistance J. Electro. Chem. Soc. $99370 \mathrm{C}$

55. Srivastav S and Kant R 2010 Theory of Generalized Cottrellian Current at Rough Electrode with Solution Resistance Effects J. Phys. Chem. C 11410066

56. Kant R, Sarathbabu M and Srivastav S 2013 Effect of Uncompensated Solution Resistance on Quasireversible 
Charge Transfer at Rough and Finite Fractal Electrode Electrochim. Acta 95237

57. Kant R and Rangarajan S K 1995 Diffusion to Rough Interfaces: Finite Charge Transfer Rates J. Electroanal. Chem. 396285

58. Harinipriya S and Sangaranarayanan M V 2001 Electron Transfer Reactions at Metal Electrodes: Influence of Work Function on Free Energy of Activation and Exchange Current Density J. Chem. Phys. 1156173

59. Limpert E, Stahel W A and Abbt M 2001 Log-normal Distributions across the Sciences: Keys and Clues BioScience. 51341

60. Aitchison J and Brown J A C 1957 In The Log-normal Distribution (Cambridge (UK): Cambridge University Press)

61. Crow E L and Shimizu K 1988 Log-normal Distributions: Theory and Application (New York: Dekker)

62. Rodgers R S and Meites L 1968 Corrections for DoubleLayer Charging in Chronopotentiometry J. Electroanal. Chem. 161
63. Isaacs H S and Kendig M W 1980 Determination of surface inhomogeneities using a scanning probe impedance technique Corrosion. 36269

64. Huang V M, Wua S L, Orazema M E, Pébèreb N, Tribollet B and Viviera V 2011 Local electrochemical impedance spectroscopy: A review and some recent developments Electrochim. Acta 568048

65. Lillard R S, Moran P J and Isaacs H S 1992 A novel method for generating quantitative local electrochemical impedance spectroscopy J. Electrochem. Soc. 1391007

66. Zou F, Thierry D and Isaacs H S 1997 A High-Resolution Probe for Localized Electrochemical Impedance Spectroscopy Measurements J. Electrochem. Soc. 144957

67. Magdić K, Kvastek K and Radošević V H 2014 Concept of spatial surface heterogeneity in impedance modelling of electrochemically activated glass-like carbon electrode Electrochim. Acta 117310

68. Lucas M and Boily J F 2015 Mapping Electrochemical Heterogeneity at Iron Oxide Surfaces: A Local Electrochemical Impedance Study Langmuir 3113618 